\title{
A method for recovering the star formation history of resolved stellar populations
}

\author{
M. Cignoni ${ }^{1,2} \dagger$, S. Degl'Innocenti ${ }^{1,3}$, P. G. Prada Moroni ${ }^{1,3}$ \\ and S. N. Shore ${ }^{1,3}$ \\ ${ }^{1}$ Dipartimento di Fisica, Università di Pisa, largo Pontecorvo 3, I-56127 Pisa, Italy \\ email: cignoni@df.unipi.it \\ ${ }^{2}$ Dipartimento di Astronomia, Università di Bologna, Via Ranzani 1, I-40127 Bologna, Italy \\ ${ }^{3}$ INFN - Sezione di Pisa, largo Pontecorvo 3, I-56127 Pisa, Italy
}

\begin{abstract}
A new method to extract information from color-magnitude diagrams (CMDs) is presented. We employ a Richardson-Lucy (RL) algorithm to the analysis of the observational uncertainties. The resulting reconstruction is a cleaned data set with which to perform analyses of the star formation history. For this task, synthetic stellar populations are built and compared with the data CMD. As an application, the star formation rate in the solar vicinity is recovered.
\end{abstract}

Keywords. Galaxy: solar neighborhood, disk, stellar content, (stars:) Hertzsprung-Russell diagram, methods: statistical

\section{Modeling the observational error}

The CMD is a key tool for studying evolution in stellar populations. However, if one wishes to infer the underlying initial mass function (IMF) and star formation rate (SFR), a careful accounting of observational uncertainties is necessary. To accomplish this, two types of approach may be used: in the first, the observational uncertainties are incorporated in the models and the data is directly compared with the modified theory. The alternative technique is to clean the data and decrease the statistical uncertainties in the observed color-magnitude diagrams. An example of the first statistical treatment is the Bayesian inference scheme proposed by Tolstoy \& Saha (1996). Here, each model point (apparent magnitude and color) is replaced with a box with a Gaussian distributed probability density (the photometric error). The total likelihood of a model is the product of the probabilities to observe the data in each box. The idea of this method is equivalent to use blurred isocrones (each point is weighted by the Gaussian spread), so that the photometric uncertainty is embodied in the theoretical model.

Following the second approach, the present work explores the possibility to eliminate the observational uncertainties directly from the data. In order to unravel the star formation history of the solar neighborhood, we explore how to obtain a cleaned input for such analyses, that is a restoration of the intrinsic distribution to the limit of the errors.

The starting-point is to treat the CMD as an image, the intensity being the number of stars in a bin ("pixel") of absolute magnitude and color. In this new framework, the observed CMD becomes a blurred image, affected by a point spread function ( $p s f$ ) that originates from the error distribution (for nearby stars the uncertainties are mainly due to parallax errors). With this as our assumption, we propose to treat it with a Richardson-Lucy algorithm. $\dagger$ Numerical experiments with artificial CMDs demonstrate

$\dagger$ Present address: Dipartimento di Astronomia, Via Ranzani 1, I-40127 Bologna, Italy

$\dagger$ This method is very well known in the astronomical community, in particular for the restoration of astronomical images (see, e.g. Bertero \& Boccacci (2005) and references therein), but this is the first time that it is applied to the study of color-magnitude diagrams. 


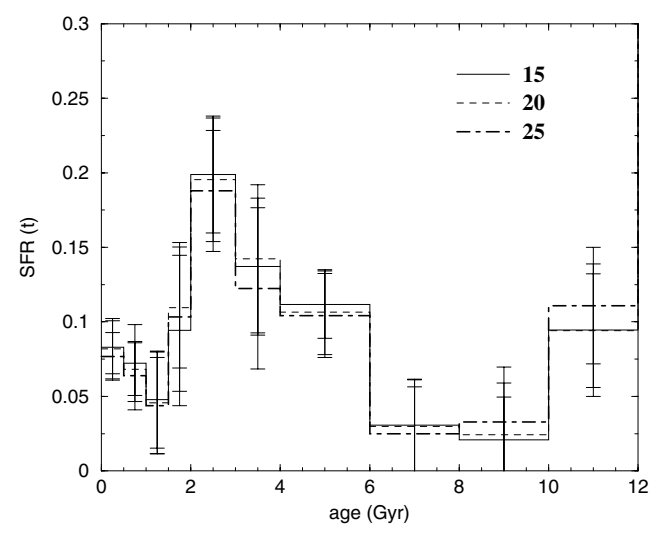

Figure 1. The recovered SFR for the solar neighborhood. Different lines show the result after the labeled number of RL iterations.

good recovery of the original image and establish convergence rates for ideal cases with single Gaussian uncertainties and Poisson noise using a $\chi^{2}$ statistic (see Cignoni \& Shore 2006 for details).

\section{Synthetic color-magnitude diagrams}

The RL restoration is applied to the local CMD ( $V$ versus $B-V$ ), built with Hipparcos stars brighter than $M_{V} \sim 3.5$ and within $80 \mathrm{pc}$. Once the CMD has been cleaned, the second step is to extract the star formation by comparing it with models. To achieve this outcome, artificial populations are created by a random choice of mass and age from the assumed IMF and SFR, interpolating on a grid of evolutionary tracks, whose metallicity is determined by the adopted age-metallicity relation (AMR). To reduce the computational time, our scheme is based on the combination of few basic synthetic CMDs (where a basic CMD is a "simple" population generated with a step star formation (uniform in a given time interval and zero elsewhere). The final CMD corresponding to any SFR is computed as a linear combination of these partial CMDs. To quantify the match between data and model CMD, a Poisson likelihood is adopted. The best SFR is searched through a simplex algorithm and the confidence limit is evaluated through a bootstrap technique.

\section{Results}

Assuming the observational AMR by Nordström et al. (2004), the resulting SFR indicates that the recent local history of the Galactic disk was very irregular (see Fig.1). The mean value increases very steeply from 6-7 Gyr ago up to 2 Gyr (see Cignoni et al. 2006), in a way qualitatively similar to the findings of Hernandez, Valls-Gabaud, \& Gilmore (2000) and Bertelli \& Nasi (2001). Recovering the local star formation has been one the possible targets of a similar technique. Indeed, general photometric surveys of other complex systems (e.g. Local Group dwarf galaxies) where the distances are well known may represent another exciting challenge for future applications.

\section{References}

Bertelli, G. \& Nasi, E. 2001, AJ, 121, 1013

Bertero, M. \& Boccacci 2005, A\&SA, 437, 369

Cignoni, M. \& S. N. Shore 2006, A\& $A, 454,511$

Cignoni, M., S. Degl'Innocenti, P. G. Prada Moroni \& S. N. Shore 2006, A\& A, 459, 783

Hernandez, X., Valls-Gabaud, D., \& Gilmore, G. 2000, MNRAS, 316, 605

Nordström, B. et al. 2004, A\&A, 418, 989

Tolstoy, E., \& Saha 1996, ApJ, 462, 672 